\title{
Quantum-Noise-Limited Optical Frequency Comb Spectroscopy
}

\author{
Aleksandra Foltynowicz, ${ }^{*}$ Ticijana Ban, ${ }^{\dagger}$ Piotr Masłowski, ${ }^{\ddagger}$ Florian Adler, ${ }^{\S}$ and Jun Ye \\ JILA, National Institute of Standards and Technology and University of Colorado, Department of Physics, \\ University of Colorado, Boulder, Colorado 80309-0440, USA
}

(Received 11 July 2011; published 28 November 2011)

\begin{abstract}
We achieve a quantum-noise-limited absorption sensitivity of $1.7 \times 10^{-12} \mathrm{~cm}^{-1}$ per spectral element at $400 \mathrm{~s}$ of acquisition time with cavity-enhanced frequency comb spectroscopy, the highest demonstrated for a comb-based technique. The system comprises a frequency comb locked to a high-finesse cavity and a fast-scanning Fourier transform spectrometer with an ultralow-noise autobalancing detector. Spectra with a signal-to-noise ratio above 1000 and a resolution of $380 \mathrm{MHz}$ are acquired within a few seconds. The measured absorption line shapes are in excellent agreement with theoretical predictions.
\end{abstract}

PACS numbers: 42.62.Fi, 33.20.-t, 42.79.Gn, 85.60.Gz

Continuous wave (cw) laser absorption spectroscopy is a well-established technique for quantitative measurements of various constituents in a gas phase. The sensitivity of the technique can be improved either by employing modulation techniques, which shift the signal to audio [1,2] or radio [3] frequencies, where the technical noise is reduced, or by implementing an external high-finesse enhancement cavity [4,5], which increases the interaction length of the light with the sample, and thus the absorption signal. A successful combination of these two approaches has led to impressive shot-noise-limited absorption sensitivities [6]. The main constraint of cavity-enhanced $\mathrm{cw}$ techniques, however, is their inability to measure broadband spectra in short acquisition times. The optical frequency combs extend the benefits of cw-laser-based techniques to thousands of laser lines and remove the bandwidth limitation $[7,8]$. The discrete spectrum of equidistant comb lines matches that of an external enhancement cavity, enabling efficient coupling of the comb light into the cavity $[9,10]$. Several detection schemes have been proposed for cavityenhanced direct frequency comb spectroscopy (CEDFCS), but none was able to suppress the technical noise down to the quantum limit. In most previous realizations of the technique, the light transmitted through the cavity has been detected using a dispersive element and a multidetector array [11-13]. With this approach the laser frequency-to-amplitude (fm-to-am) noise conversion caused by the narrow cavity modes can be efficiently reduced, by measuring either the cavity ringdown time [11] or integrated cavity output [12]. However, the sensitivity is limited by the technical noise in the detector array because of the low optical power in each spectrally resolved element. An alternative is to use Fourier transform spectrometry (FTS), either with a mechanical Michelson interferometer $[14,15]$ or with the dual comb approach [16]. The advantage of using FTS for CE-DFCS is that the spectral bandwidth of the detection system is limited only by the cavity dispersion, and not by the number of detection elements [17]. However, a photodetector and data acquisition board with a large dynamic range are necessary to obtain high signal-to-noise ratios [18]. Moreover, the use of FTS requires constant transmission through the cavity and thus the comb to be locked to the cavity, which introduces intensity noise due to fm-to-am conversion. Here we overcome these difficulties and achieve for the first time a shot-noise-limited sensitivity in CE-DFCS by tightly locking a frequency comb to a high-finesse cavity and employing a fast-scanning FT interferometer with an ultralow-noise autobalanced dual beam detection. Reaching the fundamental quantum limit makes frequency comb spectroscopy faster and more precise, which is crucial for modern applications such as medical diagnosis $[12,19]$, probing of ultracold molecules [20] or comb-based quantum information processing [21].

The system, schematically depicted in Fig. 1, is based on an Er:fiber femtosecond laser with a repetition rate of $250 \mathrm{MHz}$ and emission spectrum spanning the wavelength range from 1510 to $1610 \mathrm{~nm}$. The comb is locked by the Pound-Drever-Hall technique [22] to an external cavity with a length, $L$, of $60 \mathrm{~cm}$ and a finesse, $F$, of 8000 using error signals derived at two different wavelengths $[23,24]$. The feedback with a bandwidth of $20 \mathrm{kHz}$ is sent to the laser current and a fast piezoelectric transducer (PZT) in the laser cavity, thus stabilizing both the carrier envelope offset frequency and the comb repetition frequency to the external cavity. The details of the laser locking to the cavity are outlined in the online supplemental information [25]. The PZT correction signal is further integrated and sent to a large range PZT on which the output mirror of the external cavity is mounted to compensate for long term drifts. As a result, two (groups of) comb lines are locked to their respective cavity modes, ensuring constant transmission over hours of operation. Despite the dispersion of the cavity mirrors, up to $50 \mathrm{~nm}$ of laser spectrum can be simultaneously transmitted through the cavity, depending on the choice of the locking points. The transmitted light is analyzed with a home-built fast-scanning FT interferometer [26]. The two output beams of the interferometer are 


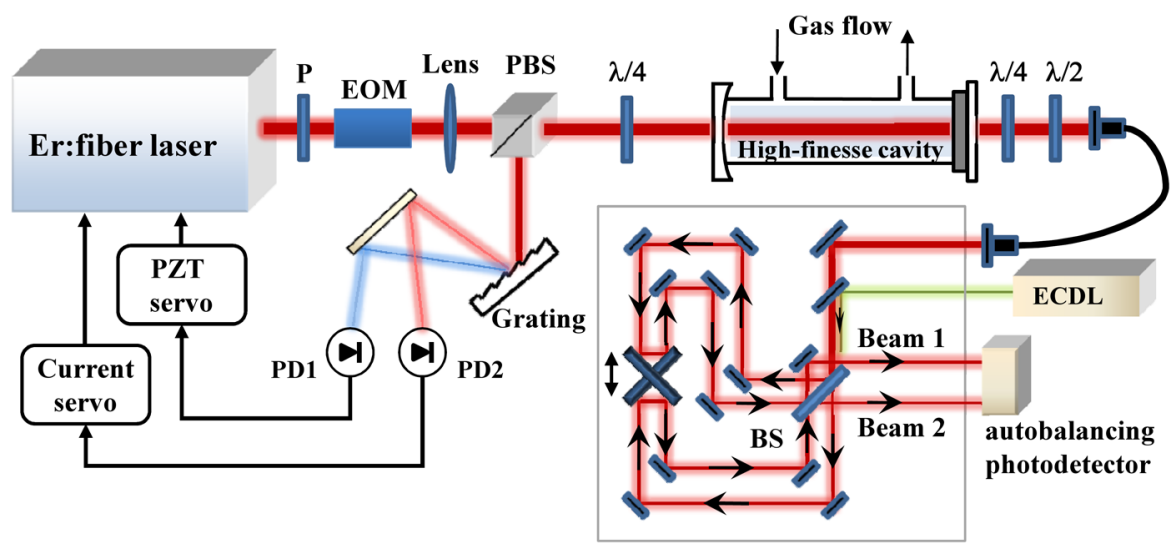

FIG. 1 (color online). Schematic of the experimental setup. Er:fiber femtosecond laser is locked to a high-finesse optical cavity containing a gas sample. An electro-optic modulator (EOM) is used to phase modulate the comb light at $14 \mathrm{MHz}$, and the cavity reflected light is dispersed by a reflection grating and imaged on two photodetectors (PD1 and PD2) in order to create error signals at two different wavelengths. The feedback is sent to laser current controller and to a PZT inside the laser cavity. The cavity transmitted light is coupled through a polarization-maintaining fiber into a fast-scanning Fourier transform spectrometer. The two outputs of the interferometer (beams 1 and 2) are incident on two photodiodes of the autobalancing photodetector. The beam of a cw $780 \mathrm{~nm}$ external cavity diode laser (ECDL), used for frequency calibration, is propagating parallel to the frequency comb beam and incident on a separate detector (not shown). $\mathrm{P}$ - polarizer, (P)BS—-(polarizing) beam splitter cube, $\lambda / 4$ — quarter wave plate, $\lambda / 2$ - half wave plate.

incident on a low-noise autobalancing InGaAs photodetector based on a Hobbs design [27], optimized to reduce the nonlinear effects in the photodiodes and transistors caused by the pulsed nature of the frequency comb. The resulting interferogram is digitized with a 22 bit data acquisition board at $1 \mathrm{Msample} / \mathrm{s}$ rate. The interferogram is then resampled at the zero crossings of a high-pass filtered interferogram of a cw $780 \mathrm{~nm}$ external cavity diode laser (ECDL), whose beam is propagating parallel to the frequency comb beam in the spectrometer.

The photocurrents from the two photodiodes in the autobalancing detector are subtracted, and the difference of the dc photocurrents is maintained at zero by a slow feedback loop. This ensures subtraction of the common mode noise [27], while the fast interferogram signal, which is out of phase in the two optical beams, is doubled. Figure 2(a) shows interferograms measured with a single photodetector (i.e., with beam 2 blocked, upper red trace) and with the autobalancing detector (lower blue trace); Fig. 2(b) shows their frequency domain representations. Because of the averaging inside the cavity, the laser intensity noise caused by the fm-to-am conversion is low pass filtered at frequencies above the cavity linewidth $\left(\sim 15 \mathrm{kHz}\right.$, corresponding to $\left.500 \mathrm{~cm}^{-1}\right)$, which is clearly visible in the spectrum displayed in red in Fig. 2(b). Proper optimization of the locking servo parameters is critical, since tighter locking results in a lower intensity noise. The high carrier frequency of the interferogram $\left(180 \mathrm{kHz}\right.$, corresponding to $6500 \mathrm{~cm}^{-1}$ ) places the laser spectrum in a region of lower noise, which is, however, still 2 orders of magnitude above the shot noise level. The dual beam autobalancing detection reduces the noise at this frequency by a factor of 600 and dramatically improves the signal-to-noise ratio in the spectrum, as shown in Fig. 2(c). We found that manual balanced detection (i.e., with the feedback in the autobalancing detector disabled) in the same interferometer provides at best noise reduction by a factor of 200 and is limited by the alignment of the two optical beams.

We evaluated the sensitivity of the system at two different spectral resolutions, namely, $380 \mathrm{MHz}$ and $2.3 \mathrm{GHz}$. For these resolutions the acquisition time, $T$, of one interferogram is $3 \mathrm{~s}$ and $0.5 \mathrm{~s}$, respectively (and twice as much for a normalized spectrum, i.e., the ratio of two consecutive background spectra measured with cavity filled with nitrogen); and the number of resolved spectral elements, $M$, is 10000 and 1500 , respectively, in a spectrum spanning $30 \mathrm{~nm}$ centered around $1530 \mathrm{~nm}$. The minimum detectable absorption in a single element of the spectrum, $\alpha_{\min }$, was calculated as the ratio of the standard deviation of the noise in the center part of a normalized spectrum and the effective interaction length inside the cavity, given by $2 F L / \pi$. Figure 3(a) shows the power dependence of the measured single element sensitivity at the two resolutions, together with the calculated shot-noise-limited sensitivity (solid lines, see online supplemental information [25]), which was confirmed by a measurement of the shot noise level on the photodetector. At a resolution of $380 \mathrm{MHz}$ [red triangular markers in Fig. 3(a)] the system is shot-noiselimited in the 30 to $100 \mu \mathrm{W}$ power range. The deviation from the shot noise limit at powers below $30 \mu \mathrm{W}$ is due to the decrease of the performance of the autobalancing detector. At powers above $100 \mu \mathrm{W}$ the intensity noise starts to dominate over the shot noise. The best shot-noiselimited single element sensitivity obtained for a $6 \mathrm{~s}$ acquisition time is $1.4 \times 10^{-9} \mathrm{~cm}^{-1}$ at $100 \mu \mathrm{W}$, corresponding 

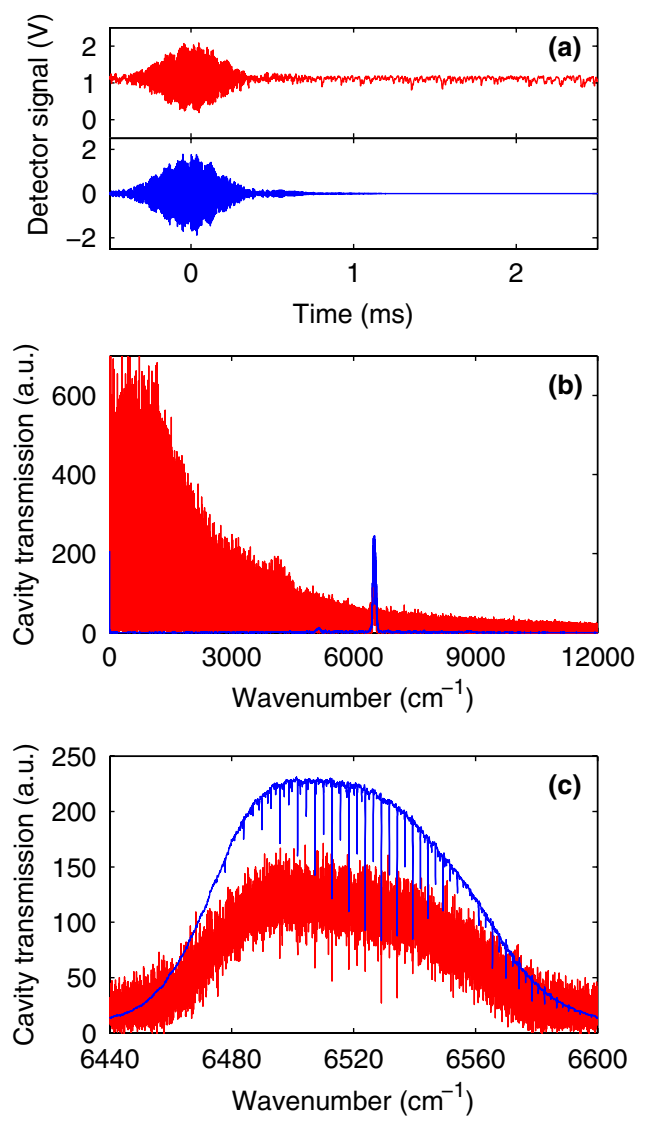

FIG. 2 (color online). (a) The centerburst of double-sided interferograms acquired in $3 \mathrm{~s}$ with one photodiode (upper red trace) and with the autobalancing detector (lower blue trace) and (b) the magnitude of their Fourier transform with a resolution of $380 \mathrm{MHz}$. The cavity was filled with $5 \mathrm{ppm}$ of acetylene in 150 Torr of nitrogen, the optical power detected on each photodiode of the autobalancing detector was $100 \mu \mathrm{W}$. The noise is reduced by a factor of 600 by the autobalancing detector at the frequency of the interferogram $(180 \mathrm{kHz}$, corresponding to $6520 \mathrm{~cm}^{-1}$ ), as is demonstrated in (c), where a zoom of (b) is shown.

to $3.4 \times 10^{-11} \mathrm{~cm}^{-1} \mathrm{~Hz}^{-1 / 2}$ per spectral element (calculated as $\alpha_{\min }(T / M)^{1 / 2}$ [26]). At a resolution of $2.3 \mathrm{GHz}$ [blue square markers in Fig. 3(a)] the measured absorption noise was slightly above that given by the shot noise limit, due to the drift of the residual etalon fringes (see online supplemental information [25]). Nevertheless, a single element sensitivity of $7.6 \times 10^{-10} \mathrm{~cm}^{-1}$ at $1 \mathrm{~s}$ was obtained, which translates to $2 \times 10^{-11} \mathrm{~cm}^{-1}$ per spectral element at $1 \mathrm{~s}$. The sensitivity of the spectrometer can be improved by optimizing the performance of the autobalancing detector at higher optical powers or by implementing a high bandwidth intensity servo in cavity transmission, as well as by eliminating the residual fringes.

To assess the long term stability of the system we measured spectra of $2 \mathrm{ppm}$ of acetylene in 150 Torr of nitrogen with a resolution of $380 \mathrm{MHz}$ and $100 \mu \mathrm{W}$ of power [such as shown in Fig. 4(a)] at equal time intervals
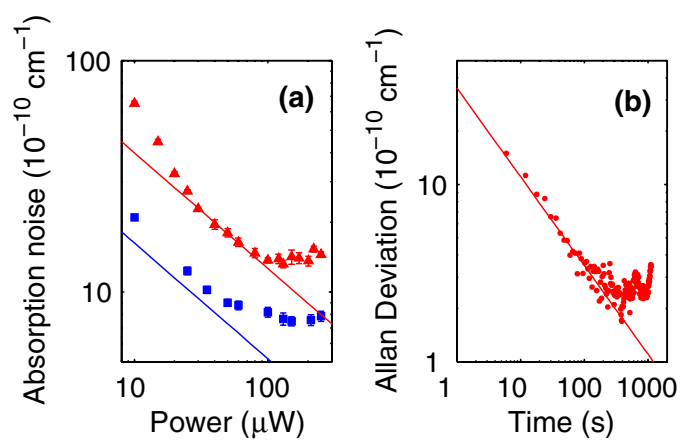

FIG. 3 (color online). (a) Single element sensitivity in a normalized spectrum as a function of optical power incident on one photodetector at a resolution of $380 \mathrm{MHz}$ (red triangular markers) and $2.3 \mathrm{GHz}$ (blue square markers). Each data point is an average of 10 and 30 measurements, respectively, and the error bars are one standard error. The red and blue lines represent the corresponding shot noise limit, which is $6^{1 / 2}$ times lower at a resolution of $2.3 \mathrm{GHz}$ because of the 6 times lower number of resolved spectral elements. (b) The Allan Deviation of a single element on the baseline between acetylene absorption lines in a spectrum measured at a resolution of $380 \mathrm{MHz}$ and $100 \mu \mathrm{W}$ of power, such as shown in Fig. 4(a). The linear fit has a $\tau^{-1 / 2}$ dependence characteristic for white noise up to $400 \mathrm{~s}$.

for $1 \mathrm{~h}$, and normalized them to a background spectrum recorded at the beginning of the measurement series. The Allan deviation of the noise on a single element of the baseline between acetylene lines in the normalized spectrum is shown in Fig. 3(b). The value of the first point of the Allan deviation (at $6 \mathrm{~s}$ ) is the same as the shot-noiselimited sensitivity at $100 \mu \mathrm{W}$ of power shown in Fig. 3(a). A linear fit with the slope of $3.5 \times 10^{-9} \mathrm{~cm}^{-1} \tau^{-1 / 2}$ [red line in Fig. 3(b)] confirms white noise behavior for averaging times, $\tau$, up to $7 \mathrm{~min}$, which means that even in the presence of an absorber the system is shot-noise-limited for long averaging times. The flicker noise floor visible at longer averaging times is due to residual etalon fringes (see [25]). The single element sensitivity at $400 \mathrm{~s}$ of averaging time is $1.7 \times 10^{-10} \mathrm{~cm}^{-1}$, corresponding to $1.7 \times 10^{-12} \mathrm{~cm}^{-1}$ per spectral element, the lowest ever reported for direct frequency comb spectroscopy [28].

Figure 4 shows experimental spectra (lower traces, in blue) of the $\nu_{1}+\nu_{3}$ band of acetylene measured with $2 \mathrm{ppm}$ of $\mathrm{C}_{2} \mathrm{H}_{2}$ in 150 Torr (panels a-c) and 630 Torr (panel d) of $\mathrm{N}_{2}$. The spectrum at the lower pressure was measured in $6 \mathrm{~s}$ with a resolution of $380 \mathrm{MHz}$, and the spectrum at atmospheric pressure was measured in only $1 \mathrm{~s}$ with $2.3 \mathrm{GHz}$ resolution, sufficient to resolve the pressurebroadened acetylene lines. The laser locking points were chosen at $1528 \mathrm{~nm}$ for the current lock and $1535 \mathrm{~nm}$ for the PZT lock. The spectra shown in red, inverted for clarity, are calculated using spectral line data from the HITRAN database [29] and a line shape model presented in the online supplemental material [25], assuming that all comb lines are on resonance with their respective cavity modes. 

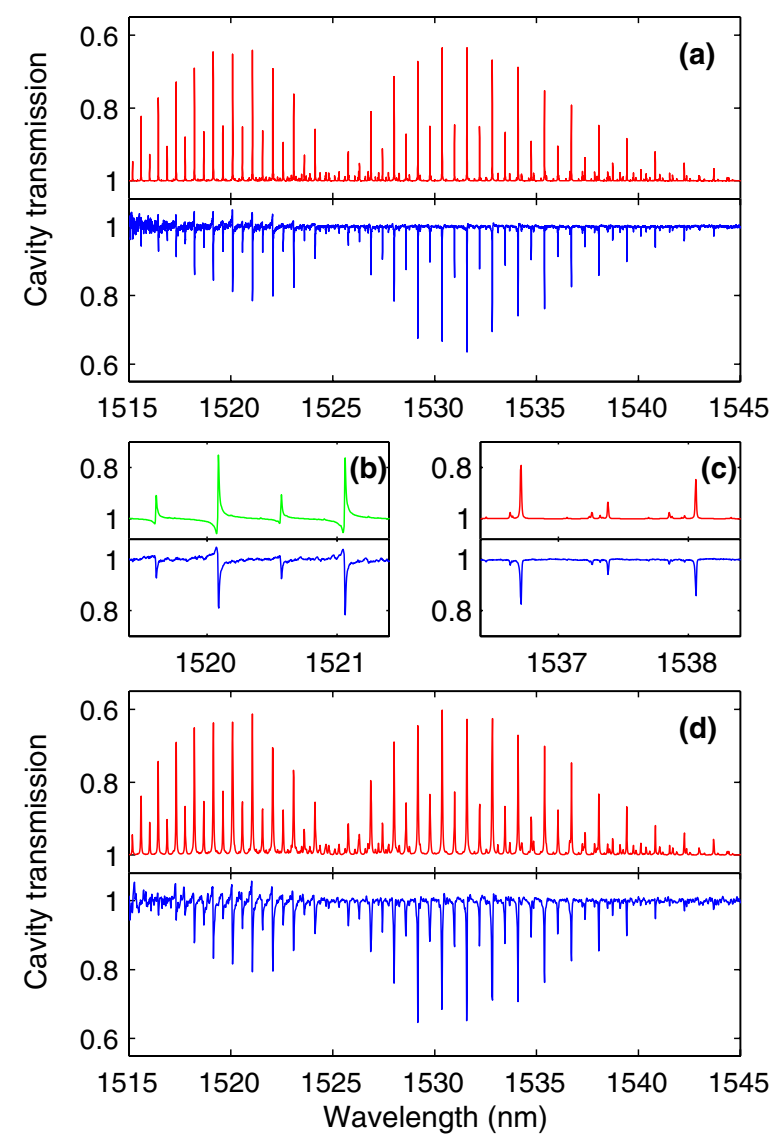

FIG. 4 (color online). Normalized experimental (blue) and theoretical (red and green, inverted for clarity) spectra of $2 \mathrm{ppm}$ of acetylene in (a)-(c) 150 Torr of $\mathrm{N}_{2}$ measured in $6 \mathrm{~s}$ with $380 \mathrm{MHz}$ resolution and in (d) 630 Torr of $\mathrm{N}_{2}$ measured in $1 \mathrm{~s}$ with $2.3 \mathrm{GHz}$ resolution. (b) and (c) show zooms of two parts of the spectrum shown in (a). (b) The slightly dispersive shape of the experimental absorption lines (blue), reproduced well in the model spectrum (green, inverted), is caused by the fact that the comb lines are offset from their respective cavity modes at wavelengths away from the laser locking points (chosen at 1528 and $1535 \mathrm{~nm}$ in this case). (c) At the locking points and in their vicinity, the comb lines are on resonance with the cavity modes and the absorption lines lack the dispersive part. The signal-to-noise ratio is lower in (b) than in (c) because of the lower comb power at these wavelengths.

A comparison of the theoretical model to the experimental data shows that the line shapes of the acetylene lines are reproduced well in the parts of the spectrum close to the locking points [see the enlarged part of the spectrum in Fig. 4(c)]. At the low wavelength edge of the spectrum (away from the locking points) dispersion of the cavity mirrors introduces a mismatch between the repetition rate of the laser and the cavity free spectral range. As a result, the comb lines are located at the slope of their respective cavity modes. In the presence of acetylene, molecular dispersion shifts the cavity modes further, causing the characteristic overshoots in the absorption line shapes, visible in the part of the spectrum enlarged in Fig. 4(b).
The theoretical spectrum, shown in green (inverted), is calculated assuming a $19 \mathrm{kHz}$ offset of the comb modes from the centers of their respective cavity modes and reproduces the observed line shapes with high accuracy. The ability to correctly model molecular line shapes is crucial for ultrasensitive trace gas detection, since neglecting the effects of intracavity dispersion would introduce systematic errors in concentration determination.

We have demonstrated for the first time cavity-enhanced direct frequency comb spectroscopy with shot-noiselimited absorption sensitivity, the highest ever achieved with a comb-based technique. High resolution broadband spectra with a signal-to-noise ratio above 1000 are acquired within seconds, and the system provides stable operation over hours. The measured absorption line shapes are in excellent agreement with theoretical model, which enables multiline fitting over the whole available spectral bandwidth for improved sensitivity and precision of trace species concentration determination [15]. The technique is thus ready for applications that demand ultrasensitive multispecies detection, such as breath analysis or detection of pollutants and hazardous gases. Implementing a midinfrared frequency comb will grant access to the strong fundamental transitions of many molecules and yield extremely low concentration detection limits. Finally, quantum-noise-limited frequency comb spectroscopy can trigger new applications of the frequency comb such as quantum information processing.

The authors thank Terry Brown for developing the autobalancing detector and Long-Sheng Ma for useful comments on the manuscript. A.F. acknowledges a Swedish Research Council fellowship; T. B. is supported through a Fulbright fellowship; and P. M. holds a fellowship from the Polish Ministry of Science and Higher Education. This project is supported by AFOSR, DTRA, NIST, NSF, and Agilent.

*aleksandra.matyba@jila.colorado.edu

${ }^{\dagger}$ Permanent address: Institute of Physics, Bijenicka Cesta 46, Zagreb, Croatia.

†Permanent address: Instytut Fizyki, Uniwersytet Mikołaja Kopernika, Torun, Poland.

${ }^{\S}$ Present address: NIST, 325 Broadway, Boulder, CO 80305, USA.

[1] H. Wahlquist, J. Chem. Phys. 35, 1708 (1961).

[2] P. Kluczynski, J. Gustafsson, A. M. Lindberg, and O. Axner, Spectrochim. Acta B 56, 1277 (2001).

[3] G. C. Bjorklund, Opt. Lett. 5, 15 (1980).

[4] A. O'Keefe and D. A. G. Deacon, Rev. Sci. Instrum. 59, 2544 (1988).

[5] B. A. Paldus and A. A. Kachanov, Can. J. Phys. 83, 975 (2005).

[6] J. Ye, L. S. Ma, and J. L. Hall, J. Opt. Soc. Am. B 15, 6 (1998). 
[7] T. Udem, R. Holzwarth, and T.W. Hansch, Nature (London) 416, 233 (2002).

[8] S. T. Cundiff and J. Ye, Rev. Mod. Phys. 75, 325 (2003).

[9] M. J. Thorpe and J. Ye, Appl. Phys. B 91, 397 (2008).

[10] F. Adler, M. J. Thorpe, K. C. Cossel, and J. Ye, Annu. Rev. Anal. Chem. 3, 175 (2010).

[11] M. J. Thorpe, K. D. Moll, R. J. Jones, B. Safdi, and J. Ye, Science 311, 1595 (2006).

[12] M. J. Thorpe, D. Balslev-Clausen, M. S. Kirchner, and J. Ye, Opt. Express 16, 2387 (2008).

[13] C. Gohle, B. Stein, A. Schliesser, T. Udem, and T. W. Hansch, Phys. Rev. Lett. 99, 263902 (2007).

[14] J. Mandon, G. Guelachvili, and N. Picque, Nat. Photon. 3, 99 (2009).

[15] F. Adler et al., Opt. Express 18, 21861 (2010).

[16] I. Coddington, W. C. Swann, and N. R. Newbury, Phys. Rev. Lett. 100, 049901(E) (2008).

[17] B. Bernhardt et al., Nat. Photon. 4, 55 (2009).

[18] N. R. Newbury, I. Coddington, and W. Swann, Opt. Express 18, 7929 (2010).
[19] T. H. Risby and S. F. Solga, Appl. Phys. B 85, 421 (2006).

[20] D. Wang et al., Phys. Rev. A 81, 061404 (2010).

[21] N. C. Menicucci, S. T. Flammia, and O. Pfister, Phys. Rev. Lett. 101, 130501 (2008).

[22] R. W. P. Drever et al., Appl. Phys. B 31, 97 (1983).

[23] R. J. Jones and J. C. Diels, Phys. Rev. Lett. 86, 3288 (2001).

[24] R. J. Jones, I. Thomann, and J. Ye, Phys. Rev. A 69, 051803 (2004).

[25] See Supplemental Material at http://link.aps.org/ supplemental/10.1103/PhysRevLett.107.233002 for details.

[26] A. Foltynowicz et al., Faraday Discuss. 150, 23 (2011).

[27] P. C. D. Hobbs, Appl. Opt. 36, 903 (1997).

[28] The sensitivity quoted in Ref. [17], which appears to be lower than that demonstrated here, is incorrectly normalized to $\mathrm{Hz}^{-1 / 2}$, as is explained in Ref. [18].

[29] L. S. Rothman et al., J. Quant. Spectrosc. Radiat. Transfer 110, 533 (2009). 